\title{
Neoadjuvant dual anti-HER2 therapy for early breast cancer: where do we stand?
}

\author{
Pankaj G. Roy ${ }^{1}$, Didier Verhoeven ${ }^{2}$ \\ ${ }^{1}$ Department of Breast Surgery, Oxford University Hospitals NHSFT, Oxford, UK; ${ }^{2}$ Department of Medical Oncology, AZ KLINA, Brasschaat, \\ University of Antwerp, Antwerp, Belgium \\ Correspondence to: Pankaj G. Roy. Consultant Oncoplastic Breast Surgeon, Department of Breast Surgery, Oxford University Hospitals NHSFT, \\ Oxford OX3 7LJ, UK. Email: pankaj.roy@ouh.nhs.uk. \\ Comment on: Shao Z, Pang D, Yang H, et al. Efficacy, safety, and tolerability of pertuzumab, trastuzumab, and docetaxel for patients with early or \\ locally advanced ERBB2-positive breast cancer in Asia: the PEONY phase 3 randomized clinical trial. JAMA Oncol 2020;6:e193692.
}

Submitted Feb 22, 2020. Accepted for publication Mar 20, 2020.

doi: $10.21037 /$ gs.2020.03.12

View this article at: http://dx.doi.org/10.21037/gs.2020.03.12

The recent published PEONY trial in the FAMA Oncology by Shao et al. reported a multicenter, double-blind, placebo-controlled randomized phase 3 trial studying the efficacy, safety and tolerability of pertuzumab along with trastuzumab and docetaxel in Asian patients with HER-2 positive non-metastatic breast cancer greater than $2 \mathrm{~cm}$ (1). Three hundred and twenty-nine patients were enrolled in the trial. Patients received 4 cycles of 3 -weekly antiHER2 therapy along with docetaxel in the neo-adjuvant setting followed by surgery followed by 3 cycles of FEC (fluorouracil, epirubicin and cyclophosphamide) and then 13 cycles of anti-HER2 therapy (similar to the one they received pre-surgery). The primary endpoint, pathologic complete response (pCR) rate demonstrated a statistically significant difference of $39.3 \%$ in the pertuzumab and trastuzumab group against a pCR of $21.8 \%$ in the placebo group (trastuzumab only). There was only a slightly higher incidence of serious adverse events in the pertuzumab group of $10.1 \%$ vs. $8.2 \%$ in the placebo group. The study design was clear and sound. pCR rate in a neo-adjuvant setting is a relevant prognostic endpoint with quick result and suggestion of a possible clinical gain.

Nevertheless, some important controversies must be assessed. pCR has been proposed as a surrogate endpoint for progression-free survival (PFS) and overall survival (OS), in trials, to expedite drug approval. It is clear from EBCTCG meta-analysis that responders (partial or complete) to systemic therapy do better than non-responders (2). The treatment in the neo-adjuvant setting helps to assess in-vivo chemosensitivity and the potential benefit of treatment. But pCR has never been validated as a trial-level surrogate for OS (3). So, the cardinal question remains if improved pCR translates into OS or PFS advantage for HER2 positive breast cancers. In the neoALTTO study, there was no difference in PFS or OS in the combination trastuzumab and lapatinib arm but patients achieving a pCR (irrespective of the treatment arm) had a better PFS and OS in comparison to those who didn't (4).

The PEONY study confirmed earlier results that the combination of chemotherapy (taxane based) with trastuzumab and pertuzumab gives more pCR in neoadjuvant setting. In the NeoSphere study, Gianni reported an increase in pCR at $45 \%$ with trastuzumab and pertuzumab vs. 29\% trastuzumab only in 2012 (5) but the follow-up NeoSphere study published in 2016 showed only a marginal benefit in the PFS after 5 years of the combination therapy over trastuzumab alone $(86 \%$ vs. $81 \%)$. In addition, the confidence intervals were wide and overlapping to achieve significance (6). Other trials such as Tryphaena cardiac safety study have addressed the superiority of dual anti-HER2 therapy in achieving a significantly higher pCR with addition of pertuzumab (7). In this study, patients who achieved pCR also had improved disease-free survival (DFS). Although the combination with pertuzumab has never been shown to increase OS, NICE recommended dual therapy in the UK for high-risk breast cancer patients with involved lymph nodes (NICE guidance TA569). 
Independent of better OS, increasing pCR could have potential advantages.

First, de-escalation of chemotherapy could be possible, avoiding anthracycline toxicity after obtaining a pCR with a taxane combination and a dual-blocker as in the PEONY trial.

Second, pertuzumab could be perhaps omitted after surgery once the pathologist has confirmed pCR. This is particularly relevant in Asian countries with limited resources and insurance coverage (8).

Third, de-escalation of surgery with a higher rate of breast conservation or even omitting surgery could become possible. A high pCR and greater than $50 \%$ correlation between complete radiological response and $\mathrm{pCR}$ has been seen in the retrospective review of 91 clinical cases audited in Oxford, suggesting a significant potential for change in surgical plan after neoadjuvant chemotherapy (9).

Another important issue is the cost-effectiveness of the addition of pertuzumab, especially in an Asian context. The addition of pertuzumab has only small clinical benefit and no proven significant survival advantage. Most big trials (NeoSphere) were not powered to provide longterm benefit (6) and others (Tryphaena and Berenice) concentrated on safety and cardiotoxicity and lacked the control arm $(7,10)$. The adjuvant Aphinity trial, reported at San Antonio Breast Cancer Symposium in 2019, that only lymph node positive patients attain a better 6-year invasive DFS with $87.9 \%$ in the pertuzumab group compared with $83.4 \%$ in the placebo group. A slightly higher incidence of toxicity, merely by a higher degree of diarrhea $38.5 \% \mathrm{vs}$. $16.4 \%$ was reported in the pertuzumab group $(11,12)$. The cost of adding pertuzumab to node negative HER2-positive patients with early breast cancer could be difficult to justify in the absence of evidence of significant long-term benefit.

Last but not least issue, is criticism on methodology, as PEONY study is industry sponsored with a major role played by the company in study design, data interpretation and writing assistance. An academic study would be more appropriate. It is also unclear if Chinese patients represent the Asian population adequately, with no patients of Indian origin included in the study.

To conclude, strategies of combining and optimizing HER2-targeted therapies could potentially improve outcomes for high-risk HER2-positive breast cancer patients but may in essence allow de-escalation of treatment in many patients, potentially sparing a lot of patients from unnecessary treatments and their related toxicities. The need for anthracyclines, number of cycles of taxane and the number of cycles of trastuzumab and pertuzumab must be assessed.

The future of HER2 patients seems anyways bright. The recent findings of additional benefit of trastuzumab emtansine (T-DM1) in the event of not obtaining a pCR in the neo-adjuvant setting (13), the introduction of trastuzumab deruxtecan (14) and in addition, promising benefit of oral HER2-selective small molecules as tucatininb (15) and neratinib would lead to further improvements in all settings but it is obligatory to critically evaluate the real benefits, certainly in the adjuvant setting.

\section{Acknowledgments}

Funding: None.

\section{Footnote}

Provenance and Peer Review: This article was commissioned by the Editorial Office, Gland Surgery. The article did not undergo external peer review.

Conflicts of Interest: Both authors have completed the ICMJE uniform disclosure form (available at http://dx.doi. org/10.21037/gs.2020.03.12). The authors have no conflicts of interest to declare.

Ethical Statement: The authors are accountable for all aspects of the work in ensuring that questions related to the accuracy or integrity of any part of the work are appropriately investigated and resolved.

Open Access Statement: This is an Open Access article distributed in accordance with the Creative Commons Attribution-NonCommercial-NoDerivs 4.0 International License (CC BY-NC-ND 4.0), which permits the noncommercial replication and distribution of the article with the strict proviso that no changes or edits are made and the original work is properly cited (including links to both the formal publication through the relevant DOI and the license). See: https://creativecommons.org/licenses/by-nc-nd/4.0/.

\section{References}

1. Shao Z, Pang D, Yang H, et al. Efficacy, safety, and tolerability of pertuzumab, trastuzumab, and docetaxel for patients with early or locally advanced ERBB2-positive breast cancer in Asia: the PEONY phase 3 randomized 
clinical trial. JAMA Oncol 2020;6:e193692.

2. Early Breast Cancer Trialists' Collaborative Group (EBCTCG). Long-term outcomes for neoadjuvant versus adjuvant chemotherapy in early breast cancer: metaanalysis of individual patient data from ten randomised trials. Lancet Oncol 2018;19:27-39.

3. Prowell TM, Beaver JA, Pazdur R. Residual disease after neoadjuvant therapy - developing drugs for high-risk early breast cancer. N Engl J Med 2019;380:612-5.

4. Huober J, Holmes E, Baselga J, et al. Survival outcomes of the NeoALTTO study (BIG 1-06): updated results of a randomised multicenter phase III neoadjuvant clinical trial in patients with HER2-positive primary breast cancer. Eur J Cancer 2019;118:169-77.

5. Gianni L, Pienkowski T, Im YH, et al. Efficacy and safety of neoadjuvant pertuzumab and trastuzumab in women with locally advanced, inflammatory, or early HER2-positive breast cancer (NeoSphere): a randomised multicentre, open-label, phase 2 trial. Lancet Oncol 2012;13:25-32.

6. Gianni L, Pienkowski T, Im YH, et al. 5-year analysis of neoadjuvant pertuzumab and trastuzumab in patients with locally advanced, inflammatory, or early-stage HER2-positive breast cancer (NeoSphere): a multicentre, open-label, phase 2 randomised trial. Lancet Oncol 2016;17:791-800.

7. Schneeweiss A, Chia S, Hickish T, et al. Longterm efficacy analysis of the randomised, phase II TRYPHAENA cardiac safety study: evaluating pertuzumab and trastuzumab plus standard neoadjuvant anthracyclinecontaining and anthracycline-free chemotherapy regimens in patients with HER2-positive early breast cancer. Eur J Cancer 2018;89:27-35.

8. Li J, Shao Z, Xu B, et al. Use of trastuzumab as an adjuvant/neoadjuvant therapy in patients with HER2positive breast cancer in China: the Nvwa study. Medicine

Cite this article as: Roy PG, Verhoeven D. Neoadjuvant dual anti-HER2 therapy for early breast cancer: where do we stand? Gland Surg 2020;9(5):1167-1169. doi: 10.21037/gs.2020.03.12
(Baltimore) 2018;97:e10350.

9. Nigam S, Hakim A, Adam R, et al. Efficacy of dual vs. single agent anti-Her-2 blockade as primary systemic therapy for Her-2 positive non-metastatic breast cancer: potential to increase likelihood of Breast Conserving Surgery. In: UK Interdisciplinary Breast Cancer Symposium 2020. Breast Cancer Res Treat 2020:abstr P052. doi: 10.1007/s10549-019-05514-3.

10. Swain SM, Ewer MS, Viale G, et al. Pertuzumab, trastuzumab, and standard anthracycline- and taxane-based chemotherapy for the neoadjuvant treatment of patients with HER2-positive localized breast cancer (BERENICE): a phase II, open-label, multicenter, multinational cardiac safety study. Ann Oncol 2018;29:646-53.

11. von Minckwitz G, Procter M, de Azambuja E, et al. Adjuvant pertuzumab and trastuzumab in early HER2positive breast cancer. N Engl J Med 2017;377:122-31.

12. Piccart M, Procter M, Fumagalli D, et al. A randomised multi-center, double-blind, placebo-controlled trial comparing chemotherapy plus trastuzumab plus pertuzumab versus chemotherapy plus trastuzumab plus placebo as adjuvant therapy in patients with operable HER2-positive early breast cancer. In: San Antonio Breast Cancer Symposium, 2019:abstr GS1-04.

13. von Minckwitz G, Huang CS, Mano MS, et al. Trastuzumab Emtansine for Residual Invasive HER2Positive Breast Cancer. N Engl J Med 2019;380:617-28.

14. Tamura K, Tsurutani J, Takahashi S, et al. Trastuzumab deruxtecan (DS-8201a) in patients with advanced HER2positive breast cancer previously treated with trastuzumab emtansine: a dose-expansion, phase 1 study. Lancet Oncol 2019;20:816-26.

15. Borges VF, Ferrario C, Aucoin N, et al. Tucatinib combined with ado-trastuzumab emtansine in advanced ERBB2/HER2-positive metastatic breast cancer: a phase 1b clinical trial. JAMA Oncol 2018;4:1214-20. 